\title{
VIM Model Test and Mooring Line Fatigue Assessment on Semi-submersible Floaters
}

\author{
by Toshifumi Fujiwara*, Member Tadashi Nimura*, Member \\ Masakatsu Saito*, Member
}

Summary

\begin{abstract}
Semi-submersible type offshore floating structures are expected to be used in the Japanese coastal area and at sea off Japan for promoting resource exploitation and development in near future. As the moored offshore floating structures are suffered from current, Vortex-induced Motion (VIM) effect should be assessed in an appropriate manner since the VIM causes fatigue damage of the floating structure's mooring lines. VIM phenomenon on semi-submersible type floating structures, however, is not clear, and its comprehension is insufficient since there are only small number of open studies with lack specifications of the structures. Then this paper represents the results of VIM measurement test using many forms of semi-submersible floating structure models to investigate the effects of column-column interval and lower hull volume for VIM amplitude, and shows the trends of VIM amplitude depending on current velocity and lower hull volume ratio for the first time. Moreover, using these results of the VIM amplitude for the models, fatigue damage of mooring lines is investigated using one sample semi-submersible offshore floating structure.
\end{abstract}

\section{Introduction}

Offshore floating rigs and structures are continuously demanded to develop oil and gas fields around the world. Semi-submersible type floating structures, i.e. semi-subs, are frequently used for drilling and production supports in the development of those fields. It is because the semi-sub's seaworthiness is superior to that of other floating bodies, e.g. ship type one, with same topside deck area in the heavy sea. A semi-sub is also used for renewable energy facilities, e.g. as a floating body for a wind power generation plant etc. for the same reason.

On the other hand, in the ISO Standard 19901-7, 'Petroleum and natural gas industries -Specific requirements for offshore structures-, Part 7: Stationkeeping systems for floating offshore structures and mobile offshore units' ${ }^{1)}$ etc., used for a general-purpose design guidance of marine structures, VIM evaluation is necessary requirement for the safety assessment of mooring lines. In the standard, it is especially noted that the VIM also occurs for semi-sub type floaters. The VIM evaluation in the design stage of the floater is needed to confirm mooring lines' safety.

The VIM phenomenon on a semi-sub is, however, reported as incomplete of information ${ }^{2 \sim 5)}$ )tc., and the technique to predict VIM amplitude depending on semi-sub specifications is not established and not recognized without model tests. VIM investigations of individual semi-subs have been conducted in the experimental tanks in each oil and gas field project, but it is hard to say that a general-purpose evaluation method of semi-sub VIM exists. Moreover, although VIM simulation of a semi-sub is tried using potential theory and CFD calculation ${ }^{6-8)}$, it seems to be difficult to calculate in short term under reliable conviction at the initial

* National Maritime Research Institute, National Institute of Maritime, Port and Aviation Technology, Japan

Received 18/May/2017
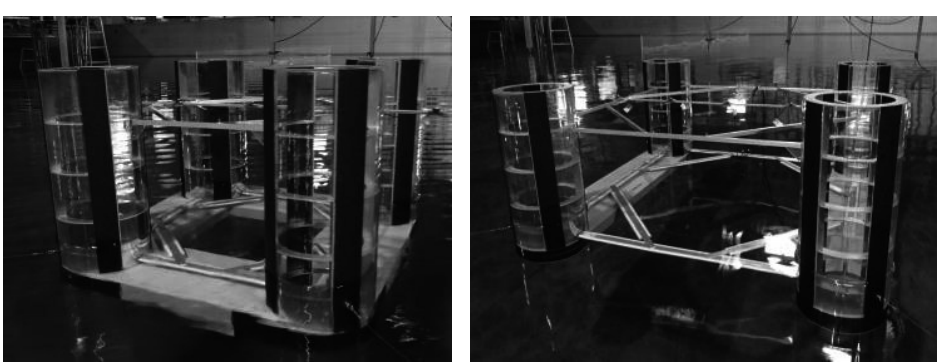

Fig. 1 Pictures of the C05d15S07 (Left) and C08d15P07 (Right).

design stage of it. Then a reasonable and simple VIM simulation method of semi-subs, which becomes effective in design sea condition, is needed from the viewpoints of the safety assessment of semi-sub operation.

In this paper, the VIM model test results on the various semi-sub models with four circular form columns with/without lower hulls are presented to understand basic VIM specification of semi-sub type floaters. Sample four columns semi-sub forms were selected from construction data ${ }^{910)}$ of semi-subs recently. VIM amplitude reduction by the effects of column-column interval and lower hull volume was clearly understood. In addition to that, simplified evaluation of the VIM amplitude of a semi-sub including lower hull volume effect was presented from the obtained model test results. The effectiveness of the evaluation method was confirmed conducting fatigue damage analysis of mooring lines of a sample semi-sub rig.

\section{VIM model tests on semi-sub floaters}

\section{1 Models}

Plural semi-sub models were prepared for the model test. Round form column was selected as the consideration of safe side from the reason that VIM seemed to be caused easily, and a four columns type semi-sub was selected as a subject from the resent semi-sub rig built trend ${ }^{910)}$. Figs. 1, 2 show the appearance of the 

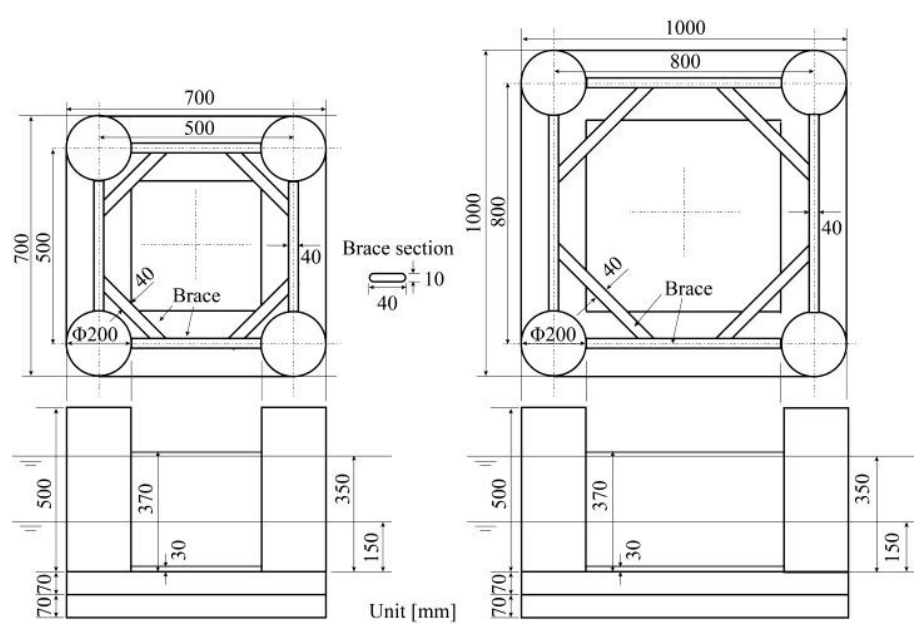

Fig. 2 Model forms on the C05 (Left) and C08 (Right) series (The pictures represent $\mathrm{S} 14$ conditions).

Table 1 Model specifications on semi-submersible floaters.

\begin{tabular}{|c|c|c|c|c|c|c|c|}
\hline Item & Unit & \multicolumn{6}{|c|}{ Semi-sub models } \\
\hline LowerHull & - & ColumnOnly & Parallel & Square & Square & ColumnOnly & Square \\
\hline Diameter (D) & $\mathrm{m}$ & \multicolumn{6}{|c|}{0.2} \\
\hline Draft (d) & $\mathrm{m}$ & 0.15 & 0.22 & 0.22 & 0.29 & 0.35 & 0.49 \\
\hline Column draft $\left(\mathrm{d}_{\mathrm{C}}\right)$ & $\mathrm{m}$ & \multicolumn{4}{|c|}{0.15} & \multicolumn{2}{|c|}{0.35} \\
\hline Lower hull height $\left(\mathrm{H}_{L H}\right)$ & $\mathrm{m}$ & 0 & 0.07 & 0.07 & 0.14 & 0 & 0.14 \\
\hline Model name & - & C05d15 & C05d15P07 & \begin{tabular}{ll|}
$\mathrm{C} 05 \mathrm{~d} 15 \mathrm{~S} 07$ \\
\end{tabular} & \begin{tabular}{|l|}
$\mathrm{C} 05 \mathrm{~d} 15 \mathrm{~S} 14$ \\
\end{tabular} & C05d35 & C05d35S14 \\
\hline Length overall (L) & $\mathrm{m}$ & \multicolumn{6}{|c|}{0.7} \\
\hline Column interval $\left(\mathrm{L}_{\mathrm{C}}\right)$ & $\mathrm{m}$ & \multicolumn{6}{|c|}{0.5} \\
\hline Mass (M) & $\mathrm{kg}$ & 19.3 & 37.7 & 46.7 & 74.1 & 44.5 & 99.2 \\
\hline Column mass $\left(\mathrm{M}_{\mathrm{C}}\right)$ & $\mathrm{kg}$ & 18.8 & 18.8 & 18.8 & 18.8 & 44.0 & 44.0 \\
\hline Lower hull mass $\left(\mathrm{M}_{\mathrm{LH}}\right)$ & $\mathrm{kg}$ & 0.5 & 18.9 & 27.9 & 55.3 & 0.5 & 55.3 \\
\hline Column ratio $\left(\mathrm{R}_{\mathrm{CLM}}\right)$ & - & 0.98 & 0.50 & 0.40 & 0.25 & 0.99 & 0.44 \\
\hline Frontal projected area $\left(\mathrm{A}_{\mathrm{WF}}\right)$ & $\mathrm{m}^{2}$ & 0.063 & 0.091 & 0.112 & 0.161 & 0.143 & 0.241 \\
\hline Lateral projected area $\left(A_{\mathrm{WL}}\right)$ & $\mathrm{m}^{2}$ & 0.063 & 0.112 & 0.112 & 0.161 & 0.143 & 0.241 \\
\hline Sway damping ratio $(\gamma)$ & $\%$ & 12.7 & 11.8 & 13.2 & 14.4 & 12.1 & 14.3 \\
\hline Sway natural period $\left(\mathrm{T}_{\mathrm{N}}\right)$ & $\mathrm{s}$ & 7.1 & 9.6 & 10.5 & 13.7 & 10.9 & 15.9 \\
\hline $\begin{array}{c}\text { Image } \\
\text { (Top and Side view) }\end{array}$ & - & $\begin{array}{ll}0 & 0 \\
0 & 0 \\
\sqcup & 4\end{array}$ & $\begin{array}{l}99 \\
\forall \forall\end{array}$ & $\begin{array}{l}09 \\
0 \quad 0 \\
41\end{array}$ & $\begin{array}{l}59 \\
60 \\
+1\end{array}$ & $\begin{array}{ll}0 & 0 \\
0 & 0 \\
& \square\end{array}$ & $\begin{array}{l}59 \\
609 \\
+11\end{array}$ \\
\hline Model name & - & C08d15 & C08d15P07 & C08d15S07 & \begin{tabular}{|l|}
$\mathrm{C} 08 \mathrm{~d} 15 \mathrm{~S} 14$ \\
\end{tabular} & & \\
\hline Length overall (L) & $\mathrm{m}$ & \multicolumn{4}{|c|}{1.0} & & \\
\hline Column interval $\left(\mathrm{L}_{\mathrm{C}}\right)$ & $\mathrm{m}$ & \multicolumn{4}{|c|}{0.8} & & \\
\hline Mass (M) & $\mathrm{kg}$ & 19.6 & 46.4 & 63.8 & 108.0 & & \\
\hline Column mass $\left(\mathrm{M}_{\mathrm{C}}\right)$ & $\mathrm{kg}$ & 18.8 & 18.8 & 18.8 & 18.8 & & \\
\hline Lower hull mass $\left(\mathrm{M}_{\mathrm{LH}}\right)$ & $\mathrm{kg}$ & 0.8 & 27.5 & 45.0 & 89.1 & & \\
\hline Column ratio $\left(\mathrm{R}_{\mathrm{CLM}}\right)$ & - & 0.96 & 0.41 & 0.30 & 0.17 & & \\
\hline Frontal projected area $\left(\mathrm{A}_{\mathrm{WF}}\right)$ & $\mathrm{m}^{2}$ & 0.066 & 0.094 & 0.136 & 0.206 & & \\
\hline Lateral projected area $\left(\mathrm{A}_{\mathrm{WL}}\right)$ & $\mathrm{m}^{2}$ & 0.066 & 0.136 & 0.136 & 0.206 & & \\
\hline Sway damping ratio $(\gamma)$ & $\%$ & 11.1 & 13.2 & 12.6 & 12.5 & & \\
\hline Sway natural period $\left(T_{N}\right)$ & $\mathrm{s}$ & 6.8 & 10.6 & 11.2 & 15.6 & & \\
\hline $\begin{array}{c}\text { Image } \\
\text { (Top and Side view) }\end{array}$ & - & \begin{tabular}{|ll}
0 & 0 \\
0 & 0 \\
& $\sqcup$
\end{tabular} & $\begin{array}{ll}9 & 9 \\
b & b \\
\forall & \forall\end{array}$ & 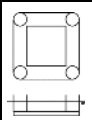 & 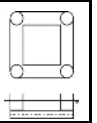 & & \\
\hline
\end{tabular}

models and Table 1 shows the model specifications. Each column has $0.2 \mathrm{~m}$ diameter, $D$. The columns were connected by oval sectioned thin braces to avoid making large drag and flow turbulence. Two types of column intervals were set using different length braces. Short brace semi-sub is named as ' $\mathrm{C} 05$ ' series with $0.5 \mathrm{~m}$ column interval, and relatively long brace ones, ' $\mathrm{C} 08$ ' series with $0.8 \mathrm{~m}$ one. Moreover, two types of lower hulls were prepared in the tests, that one was a parallel lower hull, added the character ' $\mathrm{P}$ ' in the model name, and the other was a square shaped lower hull ' $\mathrm{S}$ '. Each lower hull can be changed its thickness, 0.07 and $0.14 \mathrm{~m}$. In the tests, the draft, $d$, was also changed to investigate its effect on VIM amplitude. The column ratio, $R_{C L M}$, that has the important role to assess the VIM characteristics as mentioned later, means the ratio that total

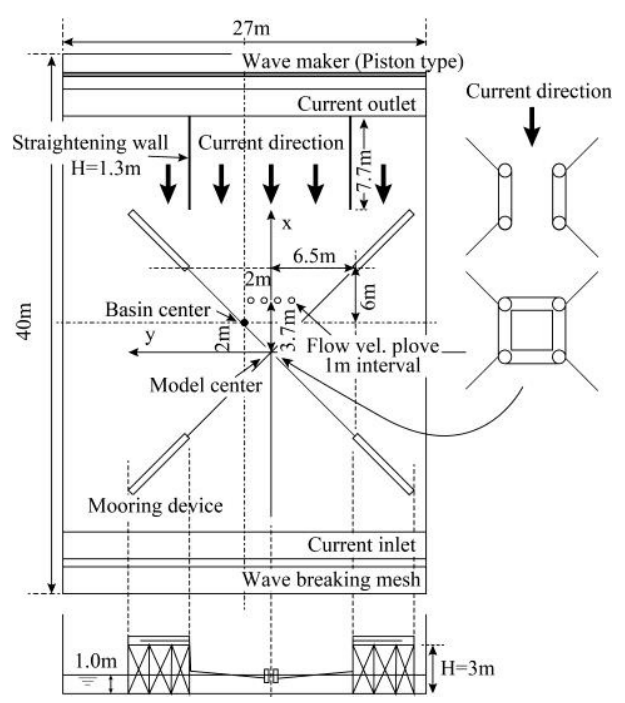

Fig. 3 Mooring condition of the model in Ocean engineering tank in NMRI (Top and Back side views).

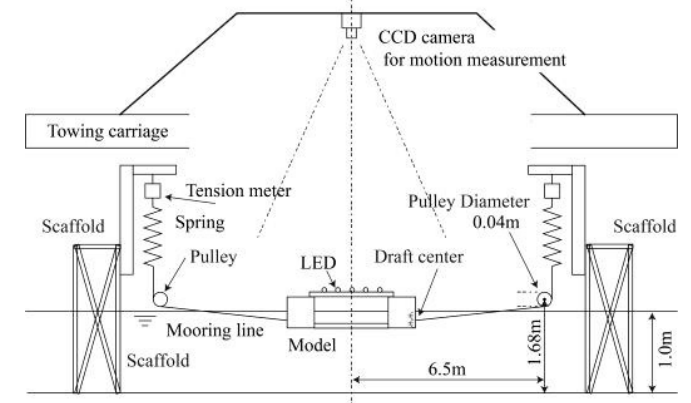

Fig. 4 Side view of mooring condition and position sensing of the model.

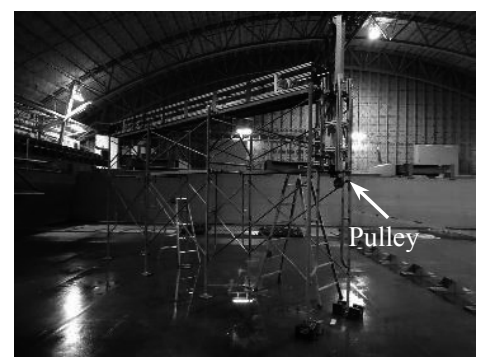

Left scaffold at upstream

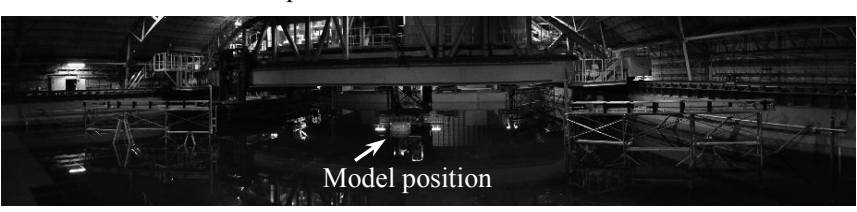

View from downstream at the tank filled with water

Fig. 5 Pictures of the experimental setting in Ocean engineering tank in NMRI.

columns displacement $V_{C}$ divided by the whole displacement $V$. The $T_{N}$ in the table is the natural period of mooring condition without current. Anti-slip tapes for turbulence enhancement, that were the 'Safety-Walk ${ }^{\mathrm{TM}} 700$ Series Coarse Tape, Black' made in $3 \mathrm{M}$ Company, $50 \mathrm{~mm}$ width ${ }^{11)}$, were stuck on four corners of the columns and the lower hulls shown in Fig. 1, that are black vertical lines etc. in the figures. 

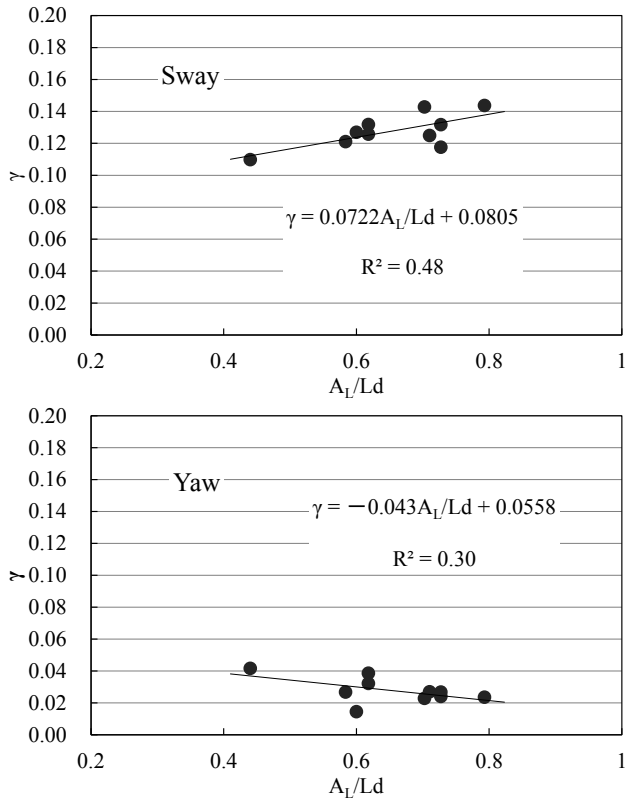

Fig. 6 Damping ratios on sway and yaw motions of the models.

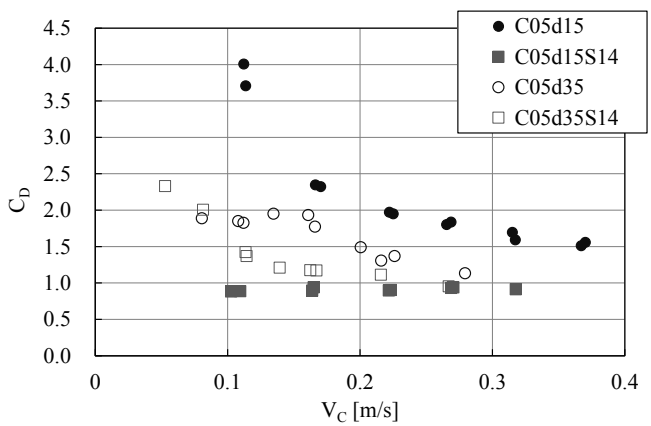

Fig. 7 Drag coefficients of the C05 series models.

\section{2 Test facility and setting}

Model test was conducted in our research institute, NMRI. Ocean engineering tank (Length $40 \mathrm{~m}$, Breadth $27 \mathrm{~m}$, Maximum water depth $2 \mathrm{~m}$ ) with a current generator was used for the test. Figs. 3, 4 and 5 show the test conditions at the tank with mooring situation. The model was set near the center of the tank floor with four thin mooring lines in the $1 \mathrm{~m}$ water depth as shown in Figs. 3, 4. Special scaffolds were prepared to set the mooring equipment. Two straightening walls were used at upstream for adjusting current flow condition.

Fig. 4 also describes mooring and position sensing arrangements of the model. A CCD camera at the top of the carriage in the tank and LED makers on the model were used to catch horizontal model position. Spring stiffness was decided from the aimed natural frequency of model oscillating displacement. As shown in Table 1, the natural period of horizontal motion in no current was set to about $7 \sim 16 \mathrm{~s}$

\section{Model test results}

\section{1 Damping quality}

As basic characteristic of the models, damping ratios of each model against critical damping are shown in Table 1 and Fig. 6. Here in the figure, the damping ratio $\gamma$ was fitted to the function $e^{-2 \pi \gamma t / T_{N}}$ using the duration time $t$, the natural
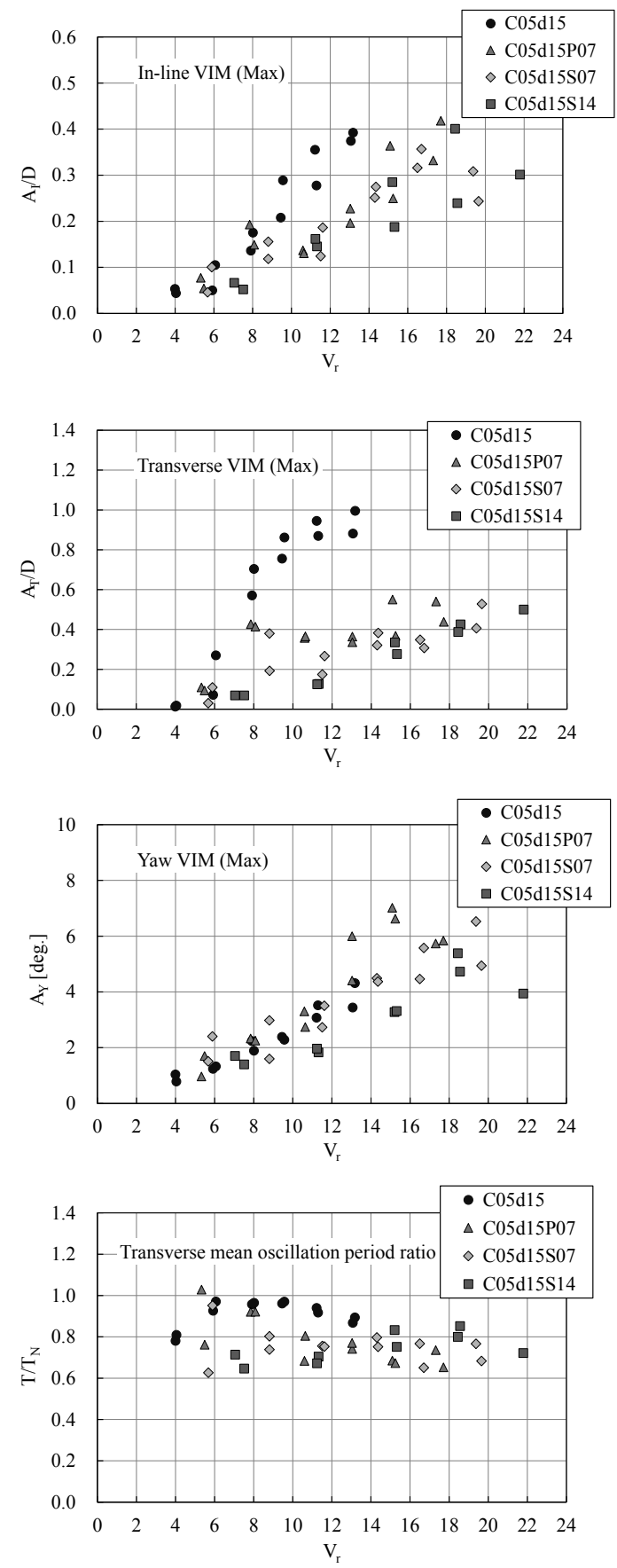

Fig. 8 Maximum amplitude ratios of the in-line, transverse and yaw VIMs of the C05 series models on lower hull effect, and transverse mean oscillation period ratios of them.

oscillation period of mooring condition $T_{N}$. The ratio of lateral projected area $A_{L} / L d$ is used as the horizontal axis in Fig. 6. The yaw dampings are considerably smaller than the sway ones. Those dampings have generally linear relation to the parameter $A_{L} / L d$.

\section{2 Drag}

Fig. 7 shows the drag coefficient of $\mathrm{C} 05$ series models as example. The definition of the drag coefficient $C_{D}$ is like this:

$$
C_{D}=\frac{F_{X}}{1 / 2 \rho_{W} A_{W F} V_{C}^{2}}
$$




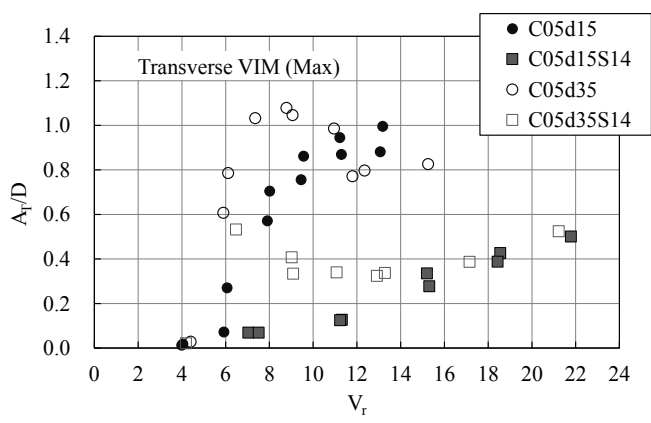

Fig. 9 Maximum amplitude ratios of the transverse VIM of the $\mathrm{C} 05$ series models on draft and lower hull effects.

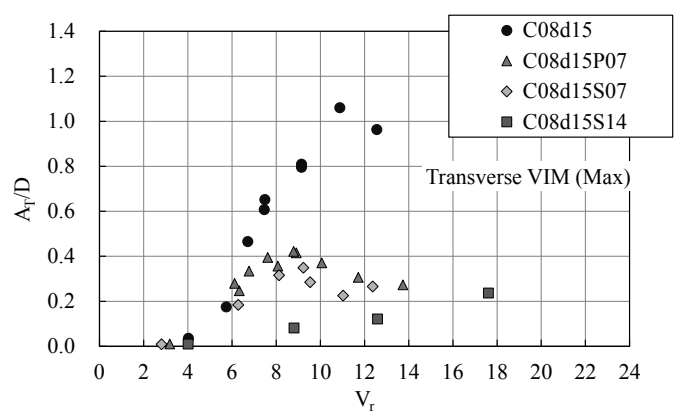

Fig. 10 Maximum amplitude ratios of the transverse VIM of the $\mathrm{C} 08$ series models on lower hull effect.

where $F_{X}$ is the averaged mooring line tension obtained from four lines, $\rho_{W}$ is the water density, $A_{W F}$ is the frontal projected area in the water, $V_{C}$ is the current velocity. The Reynolds number, $R_{e}\left(=V_{C} D / v, v\right.$ is the water kinematic viscosity coefficient), is worth for about $2 \sim 8 \times 10^{5}$. The drag forces are affected largely from lower hull conditions and current velocities.

\section{3 Model test results on VIM amplitudes}

Obtained test results are plotted using the reduced velocity $V_{r}$ in the following definition.

$$
V_{r}=V_{C} T_{N} / D
$$

The maximum values of in-line and transverse VIM ratios derived from division by the column diameter, $A_{I} / D, A_{T} / D$, are shown in Fig. 8. Moreover, the maximum yaw angles, $A_{Y}$, are shown in the same figure. As a representative, the C $05 \mathrm{~d} 15$ series are picked up at first. Here, the 'maximum' plotted data means maximum value in peak VIM amplitudes measured in one-time history test data, where averagely peak VIM amplitudes were counted about 20 30 times, that is about 200 s duration time.

The trends of the transverse VIM on semi-subs with the lower hull, added the ' $\mathrm{P}$ ' and ' $\mathrm{S}$ ' characters, are generally small values rather than ones of the C05d15. That is the reason the lower hulls seem to disturb developing vortex shedding that contributes to cause VIM. Sway damping is increasing with the increase of lower hulls volume as shown in Fig. 6. This is also the reason to reduce the VIM amplitude. The in-line VIM has also same trend relating to the transverse VIM in $V_{r} \leq 13$. In the case of yaw

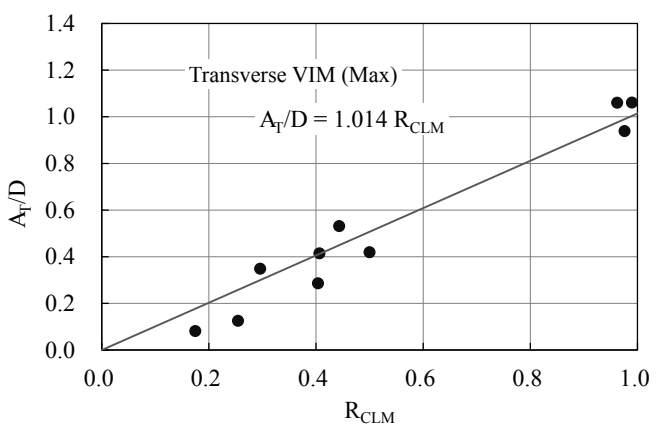

Fig. 11 Maximum amplitude ratios of the transverse VIM $\left(V_{r} \leq 15\right)$.

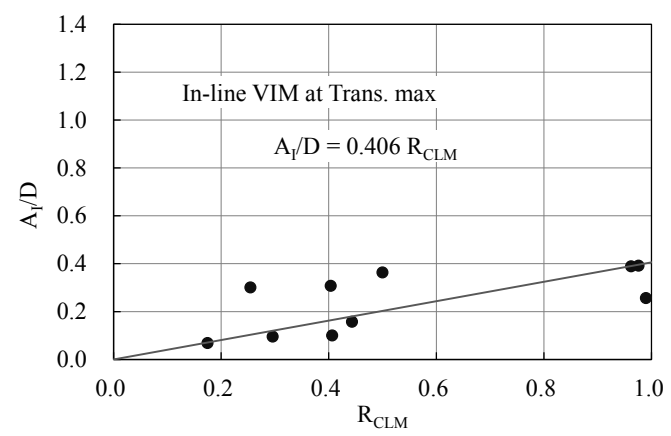

Fig. 12 In-line VIM ratios at the maximum transverse $\operatorname{VIM}\left(V_{r} \leq 15\right)$.

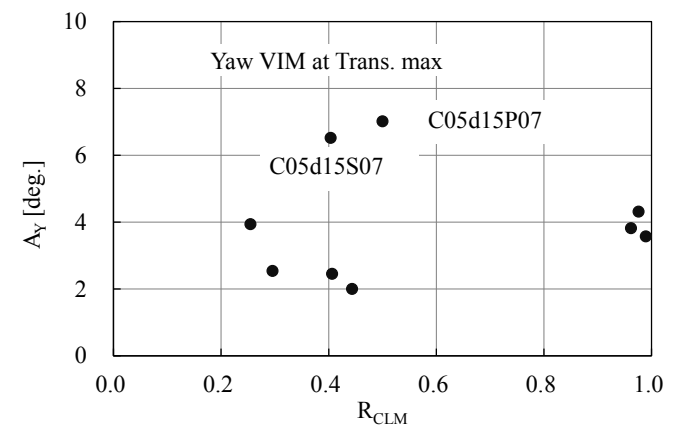

Fig. 13 Yaw VIMs at the maximum transverse VIM $\left(V_{r} \leq 15\right)$.

results, the difference of the results by the model forms is small rather than those of the in-line and transverse.

The transverse mean oscillation period ratios, $T / T_{N}$, on C05d15 are also shown in Fig. 8. Generally speaking, the mean oscillation periods have constant trend for $V_{r}$, though $10 \sim 20 \%$ of differences includes them. It is possible to say that these conditions are in lock-in phenomena. Basically, the floater VIM occurs under mooring line natural oscillation period.

Fig. 9 shows the maximum transverse VIM ratios of the C05 series models with different drafts and lower hulls. As same as Fig. 8, increasing lower hull volume causes reduction of VIM amplitude. As increasing the columns depth causes larger vortex shedding volume, the VIM amplitudes of the C05d35 and C05d35S14 at about $V_{r}=6$ become large suddenly.

Similar results in the case of the $\mathrm{C} 08$ series show in Fig. 10. The $R_{C L M}$ values of the $\mathrm{C} 08$ series are smaller than those of the C05 series. The VIM amplitudes of the C08 series seem to increase gently rather than the trend of the $\mathrm{C} 05$ series results.

It becomes clear that lower hull volume in the displacement of a semi-sub has an important role in the development of VIM 
amplitude in the current. Then using the column volume ratio in the whole displacement, $R_{C L M}$, maximum amplitude ratios of the transverse VIM in $V_{r} \leq 15$ are summarized as shown in Fig. 11, and Figs. 12, 13 show the in-line and yaw VIM amplitudes where the transverse VIMs have the maximum value. From those figures, the transverse and in-line VIMs have a linear relation for the $R_{C L M}$ generally. In contrast, the yaw VIMs seem not to have a linear trend, but the value levels of them are relatively small. It seems for present models that the yaw VIM amplitudes are smaller than $7 \mathrm{deg}$.

At the final stage, the quality of repeatability is discussed. From Figs. 8 10, generally speaking the model tests were conducted twice in the same or near current conditions. The difference of those results was about 0.1 amplitude ratio as the maximum on the in-line and transverse. The results of Figs. 11, 12 may include that level variation.

\section{Mooring line fatigue assessment}

\section{1 Sample semi-sub rig and calculation conditions}

On the basis of the previous section results, mooring line fatigue assessment is presented in this chapter. The virtual semi-sub rig with parallel lower hulls shown in Fig. 14 is treated in the assessment. This rig information was gotten from Reference 12. Mooring system was newly designed for this study.

Model specifications and calculated conditions on the sample semi-sub rig and mooring lines on fatigue damage are shown in Table 2, and the mooring lines plan and current, wind and waves directions, $\psi_{C}, \psi_{A}, \psi_{W}$, for the rig are also represented in Fig. 15. The square shape columns with round corners shown in Fig. 14 were modified to circular ones with same breadth. The mooring lines were assumed as spread taut condition. The upper ends of mooring lines were connected using some chains on the lower hull upper part. Initial tensions of the mooring lines were set to $10 \%$ of the line's MBL.

At first, mooring specifications, that is, number and diameter of lines, were decided from drift limitation of $5 \%$ in survival weather condition shown in Table 3 when $\psi_{C}, \psi_{A}, \psi_{W}=0$ deg, which detail calculation method is described in the later section. After that, mooring line fatigue assessment was done in the operation condition. Those two weather conditions shown in Table 3 and water depth were decided from Japan coastal area information ${ }^{12)}$

Constant current, wind and waves were given in the calculation. The wind and waves directions were fixed as $\psi_{A}, \psi_{W}=0 \mathrm{deg}$, and the current direction was only changed. The current was assumed to flow with the velocity of linear relation to depth of $500 \mathrm{~m}$.

\section{2 Assessment procedure for fatigue damage of mooring lines}

The recommended procedure for long-term fatigue damage assessment is shown in the regulation of $\mathrm{ISO}^{1)}$ and $\mathrm{API}^{13)}$. The calculation method conducted in this paper based on the flowchart, Fig. 16, referencing previous study ${ }^{14)}$, is shown as follows:

1) In general, some weather conditions and those durations $t$ in a year based on the probability of occurrence for that

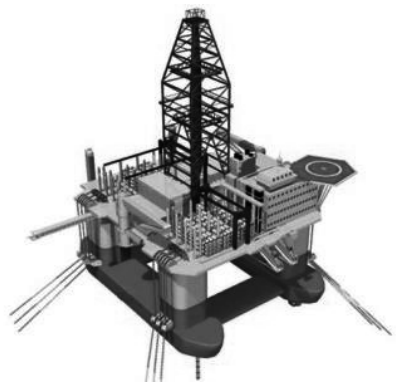

Fig. 14 Image picture of a sample semi-sub rig.

Table 2 Specifications and calculated conditions on the sample semi-sub rig and mooring lines on fatigue damage.

\begin{tabular}{ccccc}
\hline & Item & Symbol & Unit & Value \\
\hline \multirow{6}{*}{ Length } & $\mathrm{L}_{\mathrm{R}}$ & $\mathrm{m}$ & 74.4 \\
Breadth & $\mathrm{B}_{\mathrm{R}}$ & $\mathrm{m}$ & 80.0 \\
Depth & $\mathrm{D}_{\mathrm{R}}$ & $\mathrm{m}$ & 47.5 \\
& Draft (Operation) & $\mathrm{d}_{\mathrm{RO}}$ & $\mathrm{m}$ & 23.2 \\
& Draft (Survival) & $\mathrm{d}_{\mathrm{RS}}$ & $\mathrm{m}$ & 19.0 \\
& Height of column & $\mathrm{H}_{\mathrm{RC}}$ & $\mathrm{m}$ & 41.6 \\
& Breadth of column (Diameter) & $\mathrm{D}$ & $\mathrm{m}$ & 16.0 \\
& Length of lower hull & $\mathrm{L}_{\mathrm{LH}}$ & $\mathrm{m}$ & 112.0 \\
& Height of lower hull & $\mathrm{H}_{\mathrm{LH}}$ & $\mathrm{m}$ & 10.4 \\
& Breadth of lower hull (Each) & $\mathrm{B}_{\mathrm{LH}}$ & $\mathrm{m}$ & 16.0 \\
& Frontal projected area under W.L. & $\mathrm{A}_{\mathrm{WF}}$ & $\mathrm{m}$ & 742.4 \\
& Lateral projected area under W.L. & $\mathrm{A}_{\mathrm{WL}}$ & $\mathrm{m}^{2}$ & 1574.4 \\
& Frontal projected area above W.L. & $\mathrm{A}_{\mathrm{AF}}$ & $\mathrm{m}^{2}$ & 3645.0 \\
& Lateral projected area above W.L. & $\mathrm{A}_{\mathrm{AL}}$ & $\mathrm{m}^{2}$ & 3622.5 \\
& Displacement & $\mathrm{W}$ & $\mathrm{kton}$ & 50.2 \\
\hline System & - & - & Spread Taut \\
& Number of lines & - & - & 12 \\
& Material & - & - & Polyester \\
& Diameter & $\mathrm{D}_{\mathrm{ML}}$ & $\mathrm{mm}$ & 229 \\
Mooring & Length & $\mathrm{L}_{\mathrm{ML}}$ & $\mathrm{m}$ & 2037 \\
system & Mass (In air) & $\rho_{\mathrm{ML}}$ & $\mathrm{kg} / \mathrm{m}$ & 33.6 \\
& Mass (Submerged) & $\rho_{\mathrm{MLW}}$ & $\mathrm{kg} / \mathrm{m}$ & 8.4 \\
& Stiffness & $\mathrm{Stf}$ & $\mathrm{MN}$ & 397 \\
& & - & $\mathrm{kN}$ & 14715 \\
& Minimum breaking load (MBL) & - & $\mathrm{m}$ & 1500 \\
\hline & Water depth & & & \\
& & - &
\end{tabular}

W.L.: Water Line

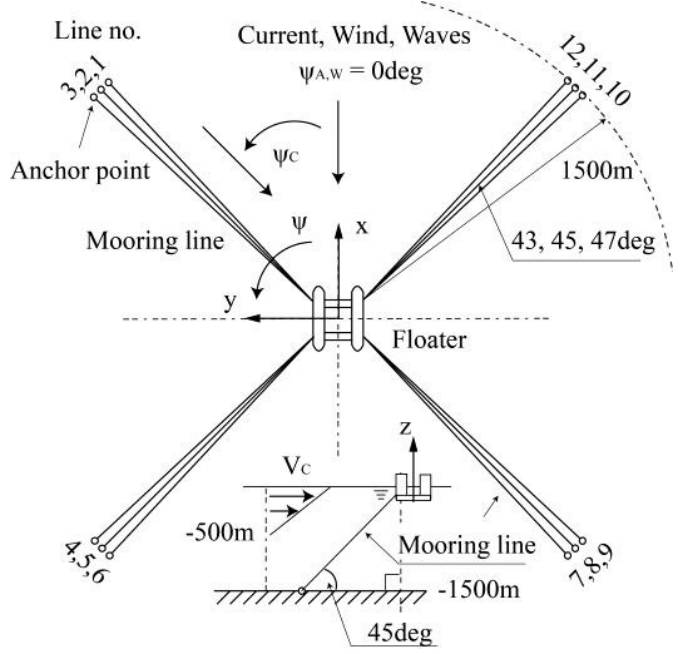

Fig. 15 Mooring lines plan and weather directions for the semi-sub rig $\left(\psi_{A}, \psi_{W}=0 \mathrm{deg}\right)$.

combination of current velocity and direction are selected. In this calculation, only one weather case shown in Table 3 was treated to know the result of most severe condition. The $t$ was, therefore, set to one year, $31,557,600 \mathrm{~s}$. This means that VIM continues for that term continuously depending on 
current velocity.

2) Determine the natural period $T_{N}$ of the moored floater under the specified weather condition without VIM. Added mass of the floater, to be necessary when calculating the $T_{N}$, was decided as same value of the floater's displacement from the model test results. Concerning floater motion, the 4-degree of freedom static equilibrium equations, excluding pitching and rolling, were used for calculation including mooring lines effect with the lumped mass concept ${ }^{15)}$. External forces with the parameters in Table 4 were obtained from following calculation formulas:

-Current

$$
\begin{gathered}
F_{C X}=\frac{1}{2} \rho_{W} A_{W F} C_{C X}\left(\psi_{C}\right) V_{C}^{2}, F_{C Y}=\frac{1}{2} \rho_{W} A_{W L} C_{C Y}\left(\psi_{C}\right) V_{C}^{2}, \\
M_{C Z}=\frac{1}{2} \rho_{W} A_{W L} L_{L H} C_{C M}\left(\psi_{C}\right) V_{C}^{2}
\end{gathered}
$$

-Wind and Waves

$F_{A X}=\frac{1}{2} \rho_{A} A_{A F} C_{A X, \psi_{A}=0} V_{A}^{2}, F_{W X}=\frac{1}{2} \rho_{W} g L_{L H} C_{W X, \psi_{W}=0}\left(\frac{H_{W}}{2}\right)^{2}$

Here, $F, M$ mean the force and yaw moment, and each suffix indicates force component and axis. $\rho_{A}$ is the air density. Force and moment coefficients on current, $C_{C}$, wind, $C_{A}$ and waves, $C_{W}$, in Table 4 getting from Reference 12 were used in the calculation.

3) Specify extreme in-line, transverse and yaw values for the current condition based on previous section model test data as shown in Table 5. As being the similar form to the sample rig, the C08d15P07 data trend was picked up. Each value of the in-line, transverse was decided using Figs. 11 and 12 by the parameter $R_{C L M}$ of the sample rig, that is equal to 0.210 . Yaw condition was assumed as the worst situation. The model test results of the VIM amplitude were represented as linear form between the given data. Namely, each parameter was connected by a straight line in $4 \leq V_{r} \leq 8$. VIM motions were assumed as following forms:

$$
\begin{aligned}
& x=A_{I} \sin \left(\frac{4 \pi}{T_{N}} t+\theta\right) \\
& {\left[\begin{array}{l}
y \\
\psi
\end{array}\right]=\left[\begin{array}{l}
A_{T} \\
A_{Y}
\end{array}\right] \sin \left(\frac{2 \pi}{T_{N}} t\right)}
\end{aligned}
$$

where $x, y$ and $\psi$ are the in-line, transverse potisions and yaw angle, respectively. $\theta$ represents phase. In the calculation, $\theta=3 / 2 \pi$ was set from the model test results. From the equilibrium position in the steady external forces, VIM motions were given in the calculation.

4) Determine drag coefficient $C_{C X}^{\prime}$ based on the transverse $A_{T} / D$ using following equation:

$$
C_{C X}^{\prime}=-0.342 \cdot A_{T} / D+C_{C X}
$$

This equation was decided from the model test data, $C_{D}$,
Table 3 Assumed weather conditions.

\begin{tabular}{ccccc}
\hline Item & Symbol & Unit & Operation & Survival \\
\hline Current velocity (Surface) & $\mathrm{V}_{\mathrm{C}}$ & $\mathrm{m} / \mathrm{s}$ & 2.2 & 2.5 \\
Wind velocity & $\mathrm{V}_{\mathrm{A}}$ & $\mathrm{m} / \mathrm{s}$ & 36.0 & 50.0 \\
Wave height & $\mathrm{H}_{\mathrm{W}}$ & $\mathrm{m}$ & 5.3 & 14.0 \\
Wave period* & $\mathrm{T}_{\mathrm{W}}$ & $\mathrm{s}$ & 11.1 & 16.2 \\
\hline
\end{tabular}

*: As a reference (no use)

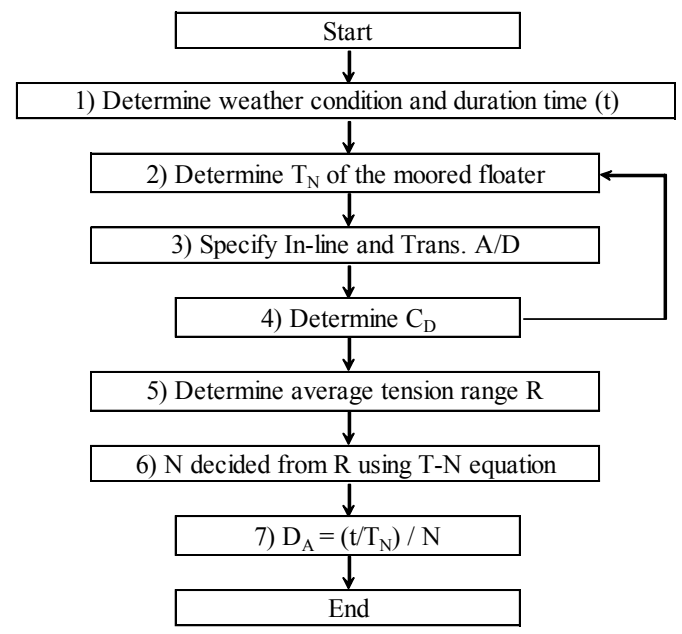

Fig. 16 Assessment procedure on long term fatigue damage of mooring lines.

Table 4 Force and moment coefficients on current, wind and waves.

\begin{tabular}{cccc}
\hline$\psi_{\mathrm{C}}$ & $\mathrm{C}_{\mathrm{CX}}$ & $\mathrm{C}_{\mathrm{CY}}$ & $\mathrm{C}_{\mathrm{CM}}$ \\
\hline 0 & -0.90 & 0.00 & 0.000 \\
15 & -1.10 & -0.60 & -0.020 \\
30 & -1.20 & -1.10 & -0.110 \\
45 & -0.85 & -1.50 & -0.124 \\
\hline$\psi_{\mathrm{A}}$ & $\mathrm{C}_{\mathrm{AX}}$ & & \\
\hline 0 & -1.0 & & \\
\cline { 1 - 2 }$\psi_{\mathrm{w}}$ & $\mathrm{C}_{\mathrm{WX}}$ & & \\
\hline 0 & -0.16 & & \\
\hline
\end{tabular}

Table 5 In-line, transverse and yaw VIM amplitudes decided from the model test data.

\begin{tabular}{ccc}
\hline$V_{r}$ & $\sim 4$ & $8 \sim$ \\
\hline$A_{I} / D$ & 0.0 & 0.085 \\
\hline$A_{T} / D$ & 0.0 & 0.213 \\
\hline$A_{Y}[$ deg. $]$ & 0.0 & 7.0 \\
\hline
\end{tabular}

shown in the previous section. If this $C_{C X}^{\prime}$ value is different from the $C_{C X}$ in Step 2, iteration is required using the $C_{C X}^{\prime}$ instead of the $C_{C X}$.

5) Perform VIM mooring analysis based on the modified VIM motion and $C_{C X}$. Determine the average maximum tension ranges $R$, and the corresponding average response period $T_{N}$ from the time trace of line tensions for VIM cycles.

6) Determine the number of cycles to failure $N$ corresponding to $R$ for the mooring component of interest using an appropriate T-N equation. A chain link, that is used at the end of mooring lines, usually has the shortest fatigue life. The 
studless chain links, Grade R5, $115 \mathrm{~mm}$ chain diameter and has same level MBL of the mooring line ${ }^{16)}$, were assumed to be equipped. The $N$ and $R$ relation of the chain is represented as follows:

$$
N R^{M}=K
$$

where $M$ is the inclination of $\mathrm{T}-\mathrm{N}$ curve, $3, K$ is the constant parameter, $316^{113)}$.

7) Calculate the annual fatigue damage:

$$
D_{A}=t /\left(T_{N} \cdot N\right)
$$

8) The predicted fatigue life is $1 / D_{A}$ years, which should be greater than the service life time considering with a safety factor. In this study, the safety factor was set as 1.0.

\section{3 Assessment results on fatigue damage of mooring lines}

Fig. 17 shows the predicted fatigue life of one of mooring lines, selected worst one, on the sample semi-sub rig in the constant weather condition. The sway natural period in mooring is about $105 \mathrm{~s}$ in the calculated current velocity range. In the strong current, where the current velocity is more than $1 \mathrm{~m} / \mathrm{s}$, fatigue life becomes shorter than 10 years by the effect of VIM. This result insists it is very important to predict the fatigue life to confirm the safety of mooring lines. The current direction effect is not so large since VIM amplitude is not changed depending on each current direction. It is effective to increase mooring tension and make the sway natural period short for putting the fatigue life off. In the case of $20 \%$ initial mooring tension, the fatigue life in $V_{C}=1 \mathrm{~m} / \mathrm{s}$ current case becomes 9 times against the $10 \%$ tension case.

When observing the details of the figure in contrast, the condition of $\psi_{C}=45 \mathrm{deg}$ has the shortest fatigue life in $V_{C}=1.4$ $\mathrm{m} / \mathrm{s}$. The line No. 12 shown in Fig. 15 has maximum tension range in the case of $\psi_{C}=45 \mathrm{deg}$ since the line No. 12 is placed at transverse VIM direction. Increasing current velocity in $\psi_{C}=45$ deg, using simplified talk, the subjected rig drifts gradually to the right direction in Fig. 15 from the reason that the lateral projected area under the water is larger than the frontal area. At that time, the tension of the line No. 12 becomes small according to the increasing drift, and the $R$ becomes large relatively. As a result, the fatigue life in the current velocity $V_{C}=1.4 \mathrm{~m} / \mathrm{s}$ is shortest value in the $\psi_{C}=45 \mathrm{deg}$ condition.

As one example, the results without yaw effect in $\psi_{C}=0 \mathrm{deg}$ are also shown in the figure. As the yaw effect can be understood clearly, it may be said that the influence of yaw should be included in the calculation according to the current situation.

Fig. 18 shows the predicted maximum tension of one of mooring lines as same as Fig. 17, and Fig. 19 shows the predicted longitudinal drift of the rig. The maximum tensions and drifts don't change largely depending on the current velocity.

The maximum value of the drift in $V_{C}=2 \mathrm{~m} / \mathrm{s}, \quad \psi_{C}=0 \sim 30 \mathrm{deg}$ is about $25 \mathrm{~m}$. This value is equivalent to $1.7 \%$ of water depth. Therefore this situation has no trouble for operation under the condition that predicted fatigue life affected from VIM effect satisfies design requirement.

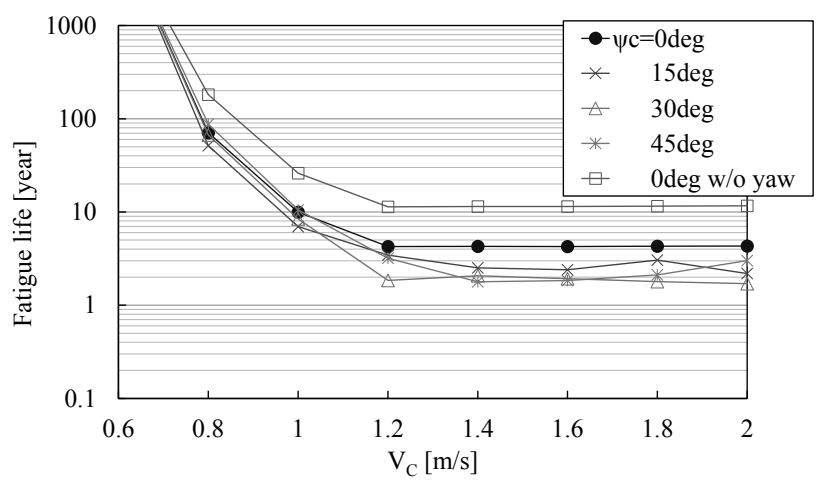

Fig. 17 Predicted fatigue lives of one of mooring lines working on maximum tension on the semi-sub in the operational sea condition.

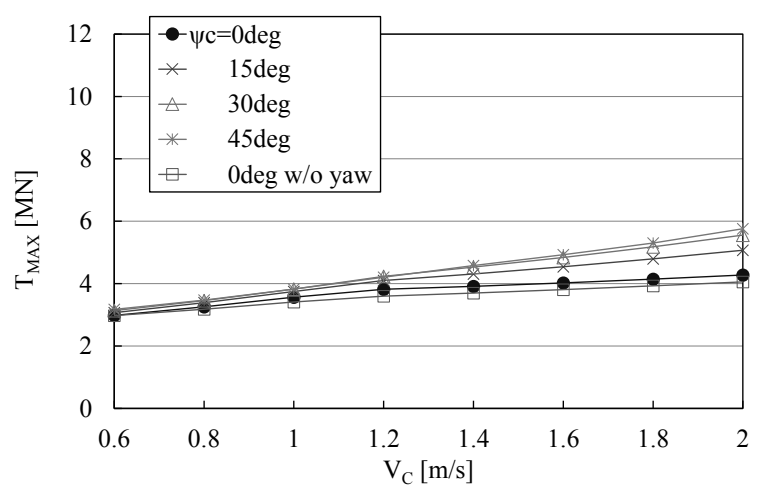

Fig. 18 Predicted maximum tensions of one of mooring lines on the semi-sub in the operational sea condition.

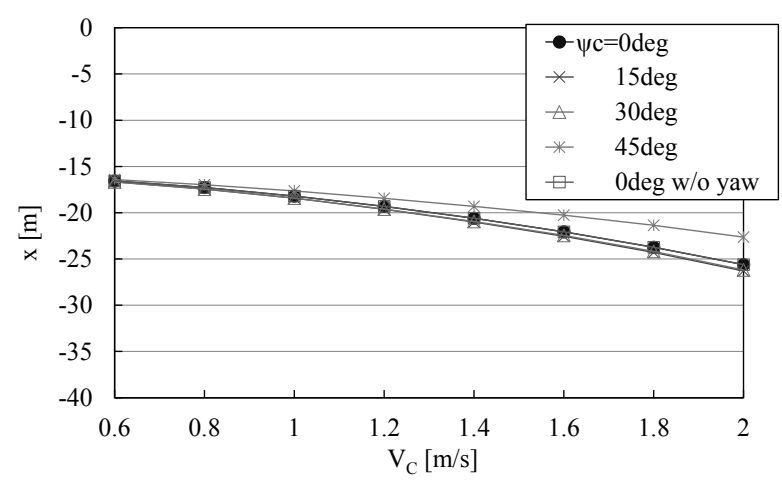

Fig. 19 Predicted longitudinal drifts of the semi-sub in the operational sea condition.

\section{Conclusions}

From the VIM model tests using various semi-sub models, the VIM interference influence between columns and lower hulls has been clarified. The maximum VIM amplitudes, that is, in-line, transverse and yaw VIMs, on the semi-subs consisting of four cylindrical columns with the combination of two kinds of column intervals and lower hull forms, have been presented as useful information. The lower hull volume influenced largely for the VIM amplitude. Then using the column ratio parameter, $R_{C L M}$, the estimation equations of VIM amplitudes on the maximum transverse and in-line were proposed for the first time in this paper. It is possible to estimate the VIM amplitude on a semi-sub 
easily by this information.

Based on these results, fatigue damage assessment of mooring lines of one sample semi-sub rig was conducted to understand the effect of the VIMs. This assessment showed the VIM effect was important for the fatigue life of mooring lines. Moreover, it was clear that the yaw effect of the semi-sub rig for the fatigue life cannot be ignored.

\section{Acknowledgments}

This research was supported by The Japan Society for the Promotion of Science (JSPS KAKENHI Grant Number 26289344). The model test was conducted with corporation by Mr. Fumitoshi Kitamura, Researcher in NMRI, Mr. Kohei Shimozato, Mitsui Engineering \& Shipbuilding Co., Ltd. (Researcher in NMRI at the time of this research) et al. The authors express deepest appreciation for them.

\section{References}

1) ISO: International Standard 19901-7 (Petroleum and natural gas industries -Specific requirements for offshore structures -, Part 7: Stationkeeping systems for floating offshore structures and mobile offshore units), 2013.

2) Waals, O. J., Phadke, A. C. and Bultema, S.: FLOW INDUCED MOTIONS OF MULTI COLUMN FLOATERS, OMAE2007-29539, 2007.

3) Gocalves, R. T., Rosetti, G. F. and Fujarra, A. L. C. et al.: EXPERIMENTAL STUDY ON VORTEX-INDUCED MOTION (VIM) OF A LARGE VOLUME SEMI-SUBMERSIBLE PLATFORM, OMAE2011-49010, 2011.

4) $\mathrm{Xu}$, Q.: A NEW SEMISUBMERSIBLE DESIGN FOR IMPROVED HEAVE MOTION, VORTEX-INDUCED MOTION AND QUAYSIDE STABILITY, OMAE2011-49118, 2011.

5) Magee, A., Sheikh, R. and Guan, K. Y. H. et al.: MODEL TESTS FOR VIM OF MULTI-COLUMN FLOATING PLATFORMS, OMAE2011-49151, 2011.

6) Bratland, A. K., Haver, S. and Stansberg, C. T. et al.: A SEMI SUBMERSIBLE IN COMBINED EXTREME WAVES AND CURRENT - COMPARISON OF MODEL TESTS AND EXISTING SOFTWARE, OMAE2007-29652, 2007.

7) Kyoung, J., Kim, J. W. and Jang, H. et al.: INVESTIGATION ON THE VIM MITIGATION OF THE HVS SEMISUBMERSIBLE, OMAE2015-41188, 2015.

8) Liu, M., Xiao, L., Lyu, H. and Tao, L.: NUMERICAL ANALYSIS OF PONTOON EFFECT ON FLOW-INDUCED FORCES OF THE DEEP DRAFT SEMISUBMERSIBLE IN A CROSS-FLOW, OMAE2015-41764, 2015.

9) Fujiwara, T., Nimura, T., Shimozato, K. and Matsui, R.: VIM MODEL TEST AND ASSESSMENT ON A SEMI-SUBMERSIBLE TYPE FLOATER, OMAE2016-54308, 2016.

10) The Mobile Offshore Drilling Units Register 2014 (15 $5^{\text {th }}$ Edition), Clarkson Research Services, 2014.

11) $3 \mathrm{M}$ Company: SafetyWalk_Tapes_Treads_July2015
LoRes.pdf, 2015.

12) Taniguchi, T., Yukawa, K., Otsubo, K. and Sato, H.: BASIC DESIGN OF A SEMI-SUBMERSIBLE RIG FOR EXPERIMENTAL DRILLING OF METHANE HYDRATE BY "HARMONIC DESIGN TOOL", OMAE2012-83309, 2012.

13) API: Recommended Practice 2 SK - Design and Analysis of Stationkeeping Systems for Floating Structures, Third Edition, 2005.

14) Fujiwara, T., Saito, M. and Maeda, K. et al.: Experimental Investigation of VIM Characteristics on Spar Type Floater in Higher Reynolds Number, Journal of the Japan Society of Naval Architects and Ocean Engineers Vol.20, pp39-47, 2014. (in Japanese)

15) Nakajima, T., Motora, S. and Fujino, M.: On the Dynamic Responses of the Moored Object and the Mooring Lines in Regular Waves, Journal of the Society of Naval Architects of Japan Vol. 150, pp.266-277, 1981. (in Japanese)

16) Class NK: 2016 Rules for the Survey and Construction of Steel Ships (Part L Chapter 1), 2016.

\section{Appendix \\ Additional information for Reynolds number and current disturbance effects on the transverse VIM amplitude}

Model Reynolds number effect and test condition effect on current disturbance for VIM amplitude were investigated to understand basic VIM characteristics for the semi-sub models. Table A1 shows the model specifications on the larger semi-sub model, C25d70, C40d70, which have five times figure forms of the $\mathrm{C} 05 \mathrm{~d} 15$ and $\mathrm{C} 08 \mathrm{~d} 15$ in addition to the small semi-subs already shown in Table 1 .

Fig. A1 shows the side view of experimental mooring condition and position sensing of the models in the Ocean engineering tank. The mooring setting for the large models is same of one shown in the References 9, 14. Water depth in the tank for large models was set to $1.5 \mathrm{~m}$ to reduce the interaction between the models and base of the tank, and in the case of the small models, C05d15 and C08d15, the water depth was set to $1.0 \mathrm{~m}$ as already shown in Fig. 4 by the reason of easy treatment

Table A1 Model specifications on large and small semi-sub models (No lower hull).

\begin{tabular}{|c|c|c|c|c|c|}
\hline Item & Unit & \multicolumn{2}{|c|}{ Large } & \multicolumn{2}{|c|}{ Small } \\
\hline Model name & - & $\mathrm{C} 25 \mathrm{~d} 70$ & C40d70 & $\mathrm{C} 05 \mathrm{~d} 15$ & $\mathrm{C} 08 \mathrm{~d} 15$ \\
\hline Diameter ratio & - & 1 & 1 & 0.2 & 0.2 \\
\hline Diameter (D) & $\mathrm{m}$ & 1.0 & 1.0 & 0.2 & 0.2 \\
\hline Draft (d) & $\mathrm{m}$ & 0.7 & 0.7 & 0.15 & 0.15 \\
\hline Column draft $\left(\mathrm{d}_{\mathrm{C}}\right)$ & $\mathrm{m}$ & 0.7 & 0.7 & 0.15 & 0.15 \\
\hline Length overall (L) & $\mathrm{m}$ & 3.5 & 5.0 & 0.7 & 1.0 \\
\hline Column interval $\left(\mathrm{L}_{\mathrm{C}}\right)$ & $\mathrm{m}$ & 2.5 & 4.0 & 0.5 & 0.8 \\
\hline Mass (M) & $\mathrm{kg}$ & 2286 & 2371 & 19.3 & 19.6 \\
\hline Column mass $\left(\mathrm{M}_{\mathrm{C}}\right)$ & $\mathrm{kg}$ & 2202 & 2202 & 18.8 & 18.8 \\
\hline Brace mass $\left(\mathrm{M}_{\mathrm{LH}}\right)$ & $\mathrm{kg}$ & 84.0 & 169.0 & 0.5 & 0.8 \\
\hline Column ratio $\left(\mathrm{R}_{\mathrm{CLM}}\right)$ & - & 0.96 & 0.93 & 0.98 & 0.96 \\
\hline Frontal and Lateral projected area $\left(\mathrm{A}_{W}\right)$ & $\mathrm{m}^{2}$ & 1.48 & 1.60 & 0.063 & 0.066 \\
\hline Sway damping ratio $(\gamma)$ & $\%$ & 20.3 & 13.5 & 12.7 & 11.1 \\
\hline Sway natural period $\left(\mathrm{T}_{\mathrm{N}}\right)$ & $\mathrm{s}$ & 30.8 & 30.6 & 7.1 & 6.8 \\
\hline $\begin{array}{c}\text { Image } \\
\text { (Top and Side view) }\end{array}$ & - & $\begin{array}{cc}0 & 0 \\
0 & 0 \\
\sqcup & \sqcup\end{array}$ & $\begin{array}{cc}0 & 0 \\
0 & 0 \\
\sqcup & \sqcup\end{array}$ & $\begin{array}{cc}0 & 0 \\
0 & 0 \\
\sqcup & \sqcup\end{array}$ & $\begin{array}{cc}\circ & 0 \\
0 & 0 \\
\square & \sqcup \\
& \sqcup\end{array}$ \\
\hline
\end{tabular}

Column ratio $\mathrm{R}_{\mathrm{CLM}}=\mathrm{V}_{\mathrm{C}} / \mathrm{V}, \mathrm{V}_{\mathrm{C}}$ : Column displaced volume, $\mathrm{V}$ : Whole displaced volume 


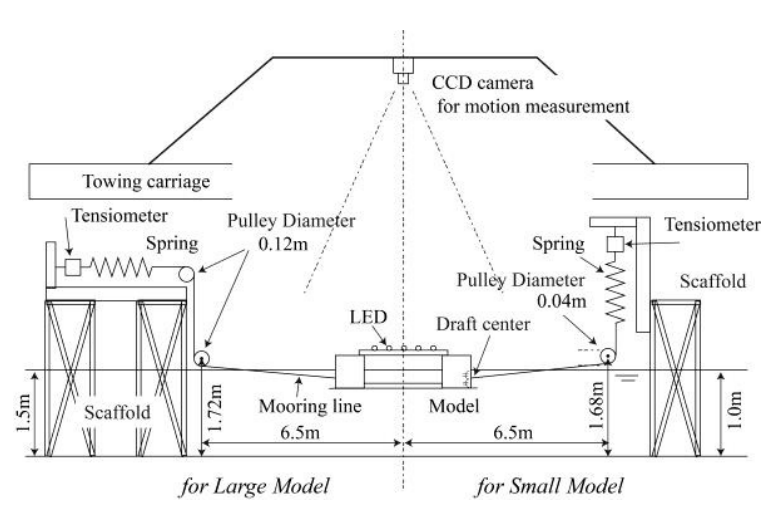

Fig. A1 Side view of the mooring condition and position sensing of the large and small models.

of model setting change.

Maximum amplitude ratios of the transverse VIM of the semi-sub models are shown in Fig. A2. The large model data obtained from different test tank in the NMRI are also added as ' $400 \mathrm{mtank}$ '. These data are the results obtained by the tank tests conducted in $400 \mathrm{~m}$ towing tank in our institute ${ }^{9)}$. The 'Ocean' data indicate the results obtained from the Ocean engineering tank shown in Figs. 3 5 and Fig. A1. Flow disturbance level in the 400 $\mathrm{m}$ towing tank is negligibly small, but at measuring points in the Ocean engineering tank shown in Fig. 3, flow velocity variances in the case of 1.0 and $1.5 \mathrm{~m}$ water depth are averagely about $10 \sim 15 \%$ and $15 \sim 25 \%$ for $0.1 \sim 0.4 \mathrm{~m} / \mathrm{s}$ velocities, respectively.

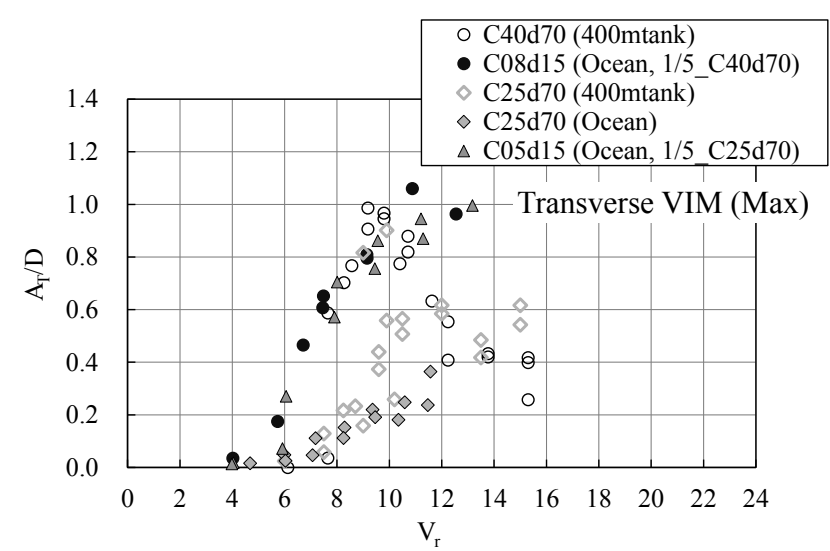

Fig. A2 Maximum amplitude ratios of the transverse VIM of the semi-sub models on difference Reynolds number and tank.

Although the large models, C25d70, C40d70, have different trend by column intervals, the interaction effect of the columns is not clear in the case of small models, C05d15, C08d15. The maximum VIM amplitudes on large and small models, however, are same level.

From the results of the $\mathrm{C} 25 \mathrm{~d} 70$, the large model seems to be affected by flow disturbance comparing the results of '400mtank' with ones of 'Ocean'.

These results represent VIM phenomenon has largely the influence of Reynolds number and flow condition. 